\title{
Méthodes diagnostiques, caractéristiques cliniques et conseil dans la mucoviscidose
}

\author{
Kris De Boeck \\ Service de Pédiatrie, Université de Louvain, Louvain, Belgique
}

\section{Mots-clés}

Mucoviscidose $\cdot$ Test de la sueur $\cdot$ Mutation du gène CFTR . Mesure de la différence de potentiel nasal - Syndrome de perte de sel $\cdot$ Pseudomonas aeruginosa $\cdot$ Insuffisance pancréatique $\cdot$ Infertilité masculine

\section{Résumé}

Au cours des premiers mois de leur vie, la plupart des patients chez lesquels une mucoviscidose sera diagnostiquée présentent des manifestations cliniques classiques de cette maladie, telles qu'un iléus méconial, un retard de la croissance staturo-pondérale et une toux persistante ou récurrente. Ces troubles sont évidents et progressifs, et affectent différents appareils et organes. Chez ces patients, l'examen diagnostique standard est le test de la sueur. Une concentration des ions chlorure supérieure à $60 \mathrm{mmol} / \mathrm{l}$ dans la sueur confirme le diagnostic cliniquement suspecté. Depuis la découverte du gène CFTR (régulateur de la conductance transmembranaire de la mucoviscidose), il est devenu évident que le spectre clinique de cette maladie s'est élargi. Des mutations du gène CFTR ont été démontrées chez plusieurs catégories de patients: patients atteints d'une mucoviscidose classique, patients présentant des troubles moins sévères, patients chez lesquels la maladie est apparue au cours de l'adolescence ou même à l'âge adulte, ou patients présentant une caractéristique clinique isolée, par exemple pan- créatite récurrente, cholangite sclérosante, bronchiectasies «idiopathiques» ou infertilité masculine. Ces deux dernières catégories ont été qualifiées de mucoviscidose atypique ou non classique. Il est très important que ces patients sachent qu'ils ne sont pas atteints d'une mucoviscidose classique, mais qu'il sont exposés au risque de maladie de type mucoviscidosique, ce qui justifie un suivi intermittent par des médecins connaissant bien le très large spectre de la maladie. Chez les patients présentant une mucoviscidose classique, des infections bactériennes sinusiennes et respiratoires chroniques et progressives avec exacerbations intermittentes sont les manifestations les plus typiques de la maladie. Une insuffisance pancréatique est présente chez $90 \%$ des patients. Les hommes sont stériles. Au cours de leur vie, les patients mucoviscidosiques peuvent présenter des manifestations pathologiques et des complications affectant les sinus, les poumons, le pancréas, le foie, l'intestin, l'œsophage, les os, les articulations, etc. Chaque patient est unique en raison de l'association complexe des manifestations possibles de la maladie. Le nombre des complications tend à s'accroître avec l'âge de ces patients, et leur état physique à s'aggraver. Une diminution de la tolérance à l'effort existe chez de nombreux adultes jeunes atteints d'une mucoviscidose en raison d'une atteinte respiratoire avancée; $20 \%$ présentent un diabète lié à la mucoviscidose. La mucoviscidose est une affection qui réduit l'espérance de vie et, même avec un traitement et un suivi des plus intensifs, la survie médiane

\section{KARGER}

Fax +4161306 1234

E-Mail karger@karger.ch

www.karger.com (c) 2006 Nestec Ltd, Vevey/S. Karger AG, Basel

0250-9644/06/0643-0119\$23.50/0

Accessible en ligne à:

www.karger.com/anf
Prof. Dr. K. De Boeck

Department of Pediatrics

Herestraat 49, BE-3000 Leuven (Belgium)

Tel. +32 16343820 , Fax +32 16343842

E-Mail christiane.deboeck@uz.kuleuven.ac.be 
actuelle est d'environ 34 ans, ce qui soumet les patients et leur famille à un stress psychologique extrêmement important. Les actions de conseil destinées à prévenir la réapparition de la maladie dans la fratrie et la famille étendue sont importantes et complexes.

Copyright $\odot 2006$ Nestec Ltd., Vevey/S. Karger AG, Basel

\section{Introduction}

La mucoviscidose est la maladie héréditaire réduisant l'espérance de vie la plus fréquente dans les populations caucasiennes. Son incidence chez les nouveau-nés varie considérablement en fonction del'ethnie de la population étudiée. Depuis la découverte du gène CFTR (régulateur de la conductance transmembranaire de la mucoviscidose), il est devenu évident que la mucoviscidose est présente dans toutes les populations étudiées à ce jour, et que son incidence a été plutôt sous-estimée chez les non-Caucasiens [1].

La mucoviscidose est une maladie héréditaire autosomique récessive due à une mutation de chacun des deux allèles du gène codant pour la protéine CFTR. Il s'agit d'une affection multiviscérale. Ses manifestations cliniques résultent d'un déficit du transport des ions chlorure à travers l'épithélium des glandes exocrines, en raison de l'absence ou du dysfonctionnement du canal chlorure CFTR activé par l'AMPc [2].

La grande majorité des patients présente des manifestations de la maladie à la naissance ou au cours des premiers mois de la vie. Le diagnostic n'est pas difficile, dans la mesure où il est envisagé et où un test de la sueur est effectué par un laboratoire expérimenté. Certains patients sont atteints d'une forme beaucoup plus discrète de la maladie, ce qui pose un problème diagnostique majeur. Ils peuvent présenter une symptomatologie variable. Chez ces patients, le résultat du test de la sueur est souvent non concluant. Des examens diagnostiques supplémentaires aident à parvenir à une conclusion: la recherche de mutations des deux allèles du gène CFTR conférant la maladie, ou des manifestations physiologiques de dysfonctionnement de la protéine CFTR [3].

\section{Signes et symptômes amenant au diagnostic de mucoviscidose}

La mucoviscidose est la maladie héréditaire grave la plus fréquente chez les Caucasiens et doit donc être souvent envisagée dans le cadre du diagnostic différentiel chez les nourrissons qui présentent des troubles récurrents ou persistants de divers types. De nombreuses manifestations cliniques peuvent être en fait les premiers signes d'une mucoviscidose.

Des vomissements et une absence d'émission de méconium surviennent chez 10 à $15 \%$ des nouveau-nés atteints d'une mucoviscidose [4]. L'iléus méconial est si spécifique de la mucoviscidose que le diagnostic est rarement ignoré. Un ictère cholestatique prolongé est une manifestation néonatale moins connue [5]. La présentation la plus fréquente est un retard de la croissance staturopondérale dû à une insuffisance pancréatique exocrine souvent associée à une toux chronique [6]. D’autre part, la digestion peut demeurer normale pendant plusieurs mois ou années [7].

Une toux récurrente ou persistante et des infections respiratoires récurrentes ou chroniques peuvent apparaître au cours de la première année de la vie et dans l'enfance. La survenue d'une toux chronique est souvent déclenchée par une infection virale, due par exemple au virus respiratoire syncytial [8].

Les syndromes de perte de sel s'observent principalement sous les climats chauds. Un coup de chaleur avec déshydratation et déséquilibre électrolytique dus à une perte excessive de sel peut aboutir au diagnostic [9]. Toutefois, même sous des climats tempérés, des nourrissons peuvent présenter une apathie, une anorexie, des vomissements, une croissance pondérale médiocre et une polyurie de type Bartter dus à une alcalose hypochlorémique et à une hypokaliémie secondaires à des apports insuffisants en sel [9].

Quand le diagnostic est ignoré au cours de la petite enfance, des complications de la maladie peuvent survenir et aboutir par la suite à son identification: hippocratisme digital, bronchiectasies, sinusite chronique, polypes nasaux, cirrhose du foie, hématémèse, hémoptysie, etc. La liste des manifestations aboutissant au diagnostic de mucoviscidose est cependant longue (tableau 1). Un souhait non satisfait de grossesse chez un couple aboutissant à la détection d'une azoospermie due à une absence bilatérale des canaux déférents chez le partenaire masculin est l'exemple classique de phénotype mucoviscidosique atypique [10; Cutting, p. 113].

Tout médecin évoquera facilement un diagnostic de mucoviscidose s'il lui est demandé quelle peut être la cause d'un retard de la croissance staturo-pondérale associé à des infections respiratoires récurrentes chez un nourrisson. Pourtant, ce diagnostic est encore souvent ignoré ou tardif, même quand la symptomatologie est classique. Les raisons de cette situation sont multiples. 
Tableau 1. Manifestations cliniques évoquant une mucoviscidose

\begin{tabular}{|c|c|}
\hline Hautement évocatrices & Évocatrices mais moins spécifiques \\
\hline \multicolumn{2}{|l|}{ Manifestations digestives } \\
\hline \multirow[t]{4}{*}{$\begin{array}{l}\text { Iléus méconial } \\
\text { Insuffisance pancréatique exocrine chez un } \\
\text { enfant }\end{array}$} & $\begin{array}{l}\text { Retard de la croissance staturo-pondérale } \\
\text { Hypoprotéinémie } \\
\text { Carences en vitamines liposolubles }\end{array}$ \\
\hline & $\begin{array}{l}\text { Syndrome d'occlusion intestinale distale } \\
\text { Prolapsus rectal }\end{array}$ \\
\hline & $\begin{array}{l}\text { Cirrhose biliaire } \\
\text { Hypertension portale } \\
\text { Lithiase biliaire chez un enfant exempt de troubles } \\
\text { hémolytiques } \\
\text { Cholangite sclérosante primitive }\end{array}$ \\
\hline & $\begin{array}{l}\text { Insuffisance pancréatique exocrine chez un adulte } \\
\text { Pancréatite récurrente }\end{array}$ \\
\hline \multicolumn{2}{|l|}{ Manifestations sinusiennes et pulmonaires } \\
\hline \multirow{2}{*}{$\begin{array}{l}\text { Infection respiratoire persistante à Pseudomonas } \\
\text { aeruginosa mucoïde } \\
\text { Bronchiectasies dans les deux lobes supérieurs } \\
\text { Infection respiratoire persistante à Burkholderia } \\
\text { cepacia }\end{array}$} & $\begin{array}{l}\text { Infections respiratoires persistantes ou récurrentes à } \\
\text { Staphylococcus aureus, P. aeruginosa, Achromobacter } \\
\text { xylosoxidans ou Haemophilus influenzae }\end{array}$ \\
\hline & $\begin{array}{l}\text { Signes radiologiques pulmonaires de bronchiectasies, } \\
\text { atélectasie, hyperinflation ou infiltrats persistants }\end{array}$ \\
\hline \multirow[t]{3}{*}{ Polypes nasaux chez un enfant } & $\begin{array}{l}\text { Hémoptysie associée à une atteinte respiratoire diffuse } \\
\text { non due à une tuberculose ou une vascularite }\end{array}$ \\
\hline & $\begin{array}{l}\text { Toux chronique et/ou productive } \\
\text { Aspergillose bronchopulmonaire allergique }\end{array}$ \\
\hline & $\begin{array}{l}\text { Polypes nasaux chez un adulte } \\
\text { Signes radiologiques de pansinusite chronique }\end{array}$ \\
\hline
\end{tabular}

\section{Autres}

Alcalose hypochlorémique en l'absence de vomissements Absence bilatérale congénitale des canaux déférents

Hippocratisme digital

Ostéopénie/ostéoporose avant l'âge de 40 ans Diabète atypique

D’après De Boeck et coll. [55] avec l'autorisation du BMJ Publishing Group.

Les parents décrivent différemment les principaux troubles. Ils ne diront pas: «Mon enfant mange beaucoup, a des selles graisseuses et ne prend pas de poids», mais plutôt: «Mon enfant est agité et difficile à calmer». En raison de la persistance des troubles, les parents peuvent consulter différents médecins plutôt que de revoir le même. Un seul trouble secondaire à une malabsorption peut retenir l'attention, plutôt que l'ensemble du tableau clinique: œdème et anémie avec envoi de l'enfant à un néphrologue pédiatre, hépatomégalie et difficultés d'alimentation motivant la consultation d'un cardiologue pédiatre, hématomes aboutissant à celle d'un cancérologue pédiatre, éruption périanale et péribuccale moti- vant celle d'un dermatologue, et anémie et hémolyse celle d'un hématologue pédiatre. Toutes ces situations ont été décrites [11]. De plus, ces enfants ne présentent fréquemment aucun épisode aigu. La toux associée à une infection bronchique chronique n'est initialement pas très intense, mais plutôt particulièrement persistante. La détresse respiratoire s'accroît lentement et progressivement. De nombreux enfants sont présentés à un médecin généraliste en raison d'infections respiratoires récurrentes. Ce médecin peut ne pas évoquer une mucoviscidose, car l'enfant semble en trop bonne santé pour cela, ou parce que sa croissance staturo-pondérale est normale. Le degré de suspicion devrait être élevé face 
à un enfant présentant des troubles chroniques. Et un test de la sueur doit être certainement réalisé chez tout enfant qui présente une expectoration ou des infiltrats pulmonaires récurrents, chez lequel la toux ne s'améliore que temporairement sous antibiothérapie, qui continue à tousser pendant l'été, chez lequel un traitement antiasthmatique est inefficace ou qui présente un hippocratisme digital.

Un diagnostic précoce est très important afin de pouvoir retarder la progression de la maladie. Un dépistage néonatal est donc recommandable dans les pays qui peuvent le financer et/ou particulièrement dans les régions où l'âge moyen est élevé au moment du diagnostic [12]. Ce dépistage n'est toutefois pas dépourvu de problèmes, car il décèlera également des hétérozygotes et des enfants chez lesquels le diagnostic est douteux [13].

\section{Examens diagnostiques spécifiques de la mucoviscidose}

Un diagnostic clinique de mucoviscidose peut être confirmé par un test de la sueur, une analyse ADN des mutations du gène CFTR ou un examen biologique de la fonction de la protéine CFTR. Lorsqu'il est disponible, le test de la sueur de Gibson et Cooke [14] est fiable, et est l'examen diagnostique initial le plus adéquat en raison de son rendement élevé: il est positif chez $98 \%$ des patients mucoviscidosiques américains [15]. L'objectif d'une analyse $\mathrm{ADN}$ «de première intention» du gène CFTR est de dépister les mutations les plus fréquentes (fréquence $>0,5 \%)$ conférant la mucoviscidose dans la population d'où le patient est originaire. Dans la plupart des populations, cet examen aboutit à la détection de mutations des deux allèles du gène chez 80 à $95 \%$ des patients [1]. Le résultat du test de la sueur est souvent ambigu chez les patients présentant des manifestations atypiques de la maladie. Des examens diagnostiques additionnels seront nécessaires afin de conforter le diagnostic: analyse des mutations du gène CFTR et, parfois, examen biologique de la fonction de la protéine CFTR.

\section{Test de la sueur}

Le rendement diagnostic d'un test de la sueur réalisé avec précision est élevé. Cet examen «simple» nécessite cependant une grande expertise et une attention méticuleuse aux détails. Dans de nombreux laboratoires, un nombre insuffisant de tests est effectué par de trop nombreux techniciens différents, et des erreurs sont donc probables [16]. Le test quantitatif de la sueur par iontopho- rèse à la pilocarpine, mis au point par Gibson et Cooke [14] en 1959, demeure l'examen de choix. Après stimulation par la pilocarpine, la sueur doit être recueillie pendant 30 minutes sur une gaze ou un papier-filtre préalablement pesé et à basse teneur en chlorure de sodium $[3$, $14,17,18]$. Un taux minimal de sudation de $1 \mathrm{~g} / \mathrm{m}^{2}$ de surface corporelle/min est nécessaire. Un "poids» de sueur de 50 à $100 \mathrm{mg}$ est donc adéquat.

Une concentration en ions chlorure supérieure à 60 $\mathrm{mmol} / \mathrm{l}$ dans la sueur confirme le diagnostic de mucoviscidose évoqué devant des manifestations cliniques. La valeur seuil de $60 \mathrm{mmol} / \mathrm{l}$ différencie de façon fiable les sujets atteints ou non d'une mucoviscidose [19]. Une concentration des ions chlorure supérieure à $60 \mathrm{mmol} / \mathrm{l}$ est presque toujours associée à une mucoviscidose. Il existe quelques exceptions: des concentrations supérieures à 60 $\mathrm{mmol} / \mathrm{l}$ ont été décrites chez des patients atteints de divers troubles cutanés peu fréquents ou d'affections endocrines ou métaboliques rares, qui peuvent être facilement distinguées d'une mucoviscidose. Même dans ce cas, il s'agit principalement de descriptions de cas isolés. La sueur normale contient moins de $60 \mathrm{mmol} / \mathrm{l}$ de chlorure et de sodium, habituellement même moins de $30 \mathrm{mmol} / \mathrm{l}$ [20]. Le test de la sueur peut être effectué dès la troisième semaine de la vie, dans la mesure où le nourrisson pèse plus de $3 \mathrm{~kg}$, est normalement hydraté et ne présente aucune affection significative. Pour des détails supplémentaires sur le test de la sueur, nous vous invitons à consulter des déclarations de consensus [3, 17, 21].

La concentration en ions chlorure dans la sueur est inférieure à $60 \mathrm{mmol} / \mathrm{l}$ chez certains sujets porteurs d'une mutation dans chacun des deux allèles du gène CFTR [22, 23]. Il n'y a pas réellement de limite inférieure absolue de la concentration en ions chlorure dans la sueur permettant d'exclure des cas atypiques de mucoviscidose [22], mais la plupart des auteurs explorent les patients chez lesquels cette concentration est de 40 à $60 \mathrm{mmol} / \mathrm{l}$. Une étude a concerné des patients dont la concentration en ions chlorure était de 30 à $60 \mathrm{mmol} / \mathrm{l}$ [20], représentant environ $4 \%$ des sujets symptomatiques ayant fait l'objet d'un test de la sueur. Des mutations des deux allèles du gène CFTR ont été décelées par la suite chez $23 \%$ de ces patients [20].

\section{Identification des mutations du gène CFTR}

Le gène $C F T R$, situé sur le bras long du chromosome 7, code pour la protéine CFTR. Cette protéine transmembranaire agit comme un canal chlorure dépendant de l'AMPc et exerce de nombreuses fonctions, dont l'une des plus importantes est la régulation de l'équilibre hydro- 
électrolytique à travers les épithéliums [2; Donaldson et Boucher, pp. 103-109].

Plus de 1500 mutations du gène CFTR ont été identifiées [24], et plus de 1000 ont été décrites chez des patients présentant des manifestations de mucoviscidose. Les mutations restantes induisent les formes moins sévères de mucoviscidose, ou ne confèrent pas la maladie [24]. Plusieurs mutations (la plupart des mutations de classe I: mutations non-sens ou de site d'épissage, insertions/délétions hors du cadre de lecture) sont manifestement à l'origine d'une mucoviscidose. Seules des études fonctionnelles permettent de déterminer sans équivoque si les mutations restantes confèrent cette maladie. A ce jour, ces études n'ont été réalisées que pour les mutations les plus fréquentes du gène CFTR. Une rare mutation fauxsens décelée après un criblage complet du gène CFTR peut être soit un polymorphisme, soit une mutation à l'origine d'une mucoviscidose [Cutting, p. 113].

La mucoviscidose est une maladie autosomique récessive, ce qui signifie qu'une mutation pathogène affectant les deux allèles du gène CFTR est présente chez les patients atteints. Un patient mucoviscidosique peut être homozygote si les mutations des deux allèles de CFTR sont identiques, ou hétérozygote composite si elles sont différentes.

La fréquence des mutations de CFTR diffère selon les populations [1]. F508del est de loin la mutation la plus fréquente et est retrouvée sur $70 \%$ ou plus des chromosomes des patients mucoviscidosiques dans les populations de l'Europe du nord. Les fréquences de F508del sont beaucoup plus faibles dans les populations d'Europe du sud. Des exemples d'autres mutations pouvant survenir dans la plupart des populations avec une fréquence d'environ $1-2 \%$ sont G542X, G551D, R553X, W1282X et N1303K. Enfin, dans une population donnée, des mutations ethnospécifiques peuvent atteindre une fréquence de $1 \%$, ou même de $7 \%$ [25]. Dans la plupart des populations, ces mutations fréquentes concernent $80-95 \%$ de l'ensemble des gènes CFTR mutants.

Des méthodes commercialisées sont disponibles pour la recherche simultanée d'environ 30 mutations dont la prévalence est supérieure à $0,5 \%$ et dont la majorité est associée à une mucoviscidose classique [26]. Un taux de détection de mutations de $90 \%$ dans une population spécifique signifie qu'une mutation des deux allèles du gène CFTR sera identifiée chez $81 \%$ des patients atteints d'une mucoviscidose typique; une mutation ne le sera que sur un seul de ces allèles chez $18 \%$, et aucune ne le sera sur l'un ou l'autre chez $1 \%$.

En cas de résultat limite du test de la sueur chez un patient présentant des troubles compatibles avec une mu- coviscidose atypique, une recherche étendue de mutations des deux allèles du gène CFTR peut être nécessaire afin de conforter ce diagnostic. Seul un séquençage approchera une sensibilité de $100 \%$. Même quand une mutation est décelée, son rôle causal dans la maladie peut ne pas être clair: les conséquences fonctionnelles de nombreuses mutations du gène CFTR sont inconnues; il peut s'agir de polymorphismes [24].

\section{Examens biologiques de la protéine CFTR: \\ Différence de potentiel transépithélial nasal}

La mucoviscidose est une maladie due à l'absence ou au dysfonctionnement de la protéine CFTR apicale responsable d'une sécrétion de chlorure induite par l'AMPc. Il existe de plus un afflux compensateur excessif de sodium dans les cellules épithéliales [2,27]. Les examens biologiques de CFTR mesurent les flux ioniques épithéliaux ou les différences de potentiel qui en résultent à la surface de la muqueuse [28]. Ces méthodes donnent ainsi une représentation directe de la physiologie au niveau des cellules et des canaux ioniques et ont été développées afin de faciliter la résolution de dilemmes diagnostiques chez des patients atypiques, en permettant d'exclure ou de confirmer un dysfonctionnement lié à la protéine CFTR. Ces examens peuvent être réalisés sur l'épithélium respiratoire ou intestinal. Ils sont complexes et nécessitent un équipement spécialisé et une expertise particulière. L'utilisation de la différence de potentiel (ddp) nasal à titre de méthode diagnostique dans la mucoviscidose avait été déjà acceptée dans le consensus diagnostique de Rosenstein et Cutting [29] en 1998. Cette ddp est mesurée entre la muqueuse nasale et la peau de l'avantbras. Des détails pratiques sur cet examen ont été publiés $[28,30]$. La ddp basale donne une indication du transport du sodium par le canal épithélial sodique sensible à l'amiloride, L'effet du blocage de ce canal par une application d'amiloride est alors évalué. Enfin, un important gradient chimique du chlorure est produit à travers la membrane apicale afin de mesurer la perméabilité épithéliale à cet ion.

Cet examen a été introduit en pratique clinique par de nombreux centres importants de traitement de la mucoviscidose, qui ont compilé leurs propres valeurs de référence. Avec des opérateurs entraînés et une étroite attention portée aux détails techniques, la mesure de la ddp nasale peut être normalisée [31].

La ddp nasale d'un patient atteint d'une mucoviscidose classique est facilement différenciable de celle d'un sujet témoin en bonne santé (fig. 1). Chez un patient atteint d'une mucoviscidose, la ddp basale est plus négative, 
Fig. 1. Mesure de la différence de potentiel (ddp) nasale chez un sujet en bonne santé (a) et un sujet atteint d'une mucoviscidose typique (b). Chez un sujet en bonne santé, la ddp basale est négative ( -20 à $30 \mathrm{mV})$, augmente modérément après une application d'amiloride et diminue très nettement après celle d'une solution à faible teneur en chlorure et d'isoprénaline. Chez un patient atteint d'une mucoviscidose typique, la ddp basale est plus négative, et l'augmentation est plus importante après application d'amiloride. La ddp ne varie pas après celle d'une solution à faible teneur en chlorure et d'isoprénaline. D'après De Boeck et coll. [55] avec l'autorisation du BMJ Publishing Group.
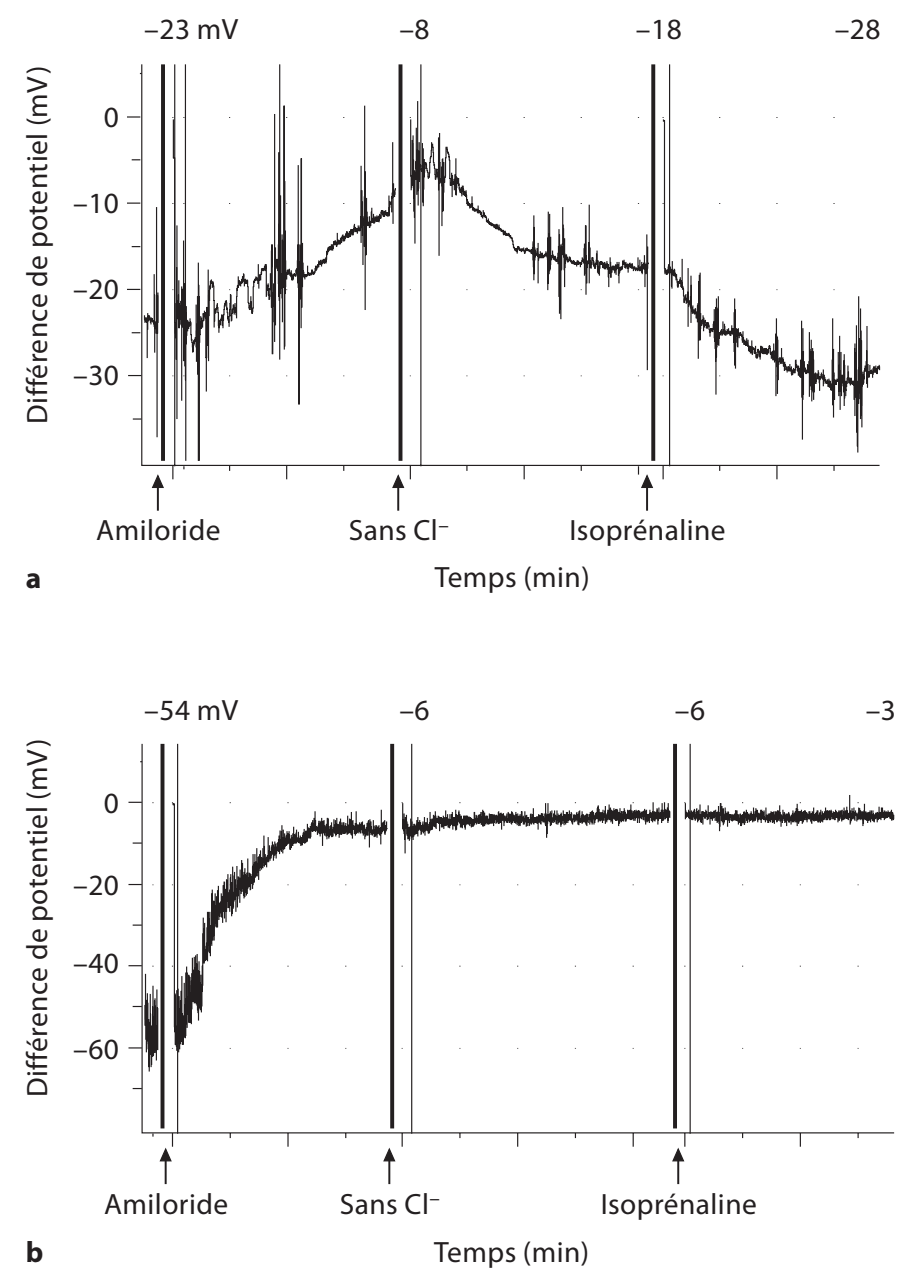

la réponse à l'amiloride est plus importante et la réponse à une solution sans chlorure et à l'isoprénaline est faible ou absente. Dans la mucoviscidose non classique, la valeur de la ddp nasale peut être limite, et il n'existe encore aucun consensus quant à ce qui constitue exactement un résultat anormal.

\section{Aspects cliniques}

Comme mentionné plus haut, la mucoviscidose est une maladie multiviscérale. Ses manifestations cliniques caractéristiques sont des infections sinusiennes et respiratoires chroniques, des anomalies digestives et nutri- tionnelles, un syndrome de perte de sel et des anomalies de l'appareil génital masculin aboutissant à une azoospermie obstructive (tableau 1). La quasi-totalité des patients présentera une atteinte sinusienne et respiratoire chronique. Une insuffisance pancréatique est présente chez $90 \%$ des patients. Les hommes sont stériles.

\section{Atteinte respiratoire basse}

Une infection et une inflammation respiratoires chroniques avec exacerbations intermittentes sont le principal problème et limitent la durée et la qualité de la vie de la plupart des patients mucoviscidosiques. Des infections respiratoires bactériennes associées à un accroissement de la réponse inflammatoire de l'hôte surviennent dès le 
début de la vie [32]. Un déséquilibre hydroélectrolytique dans les voies aériennes aboutit à des sécrétions visqueuses et à une obstruction. Cette situation contribue fortement à la pérennisation de l'infection et de l'inflammation $[2,27]$. Le lien exact entre le dysfonctionnement de la protéine CFTR et l'infection bactérienne chronique n'est cependant pas totalement élucidé [Donaldson et Boucher, pp. 103-109]. Les bactéries les plus typiquement responsables d'infections respiratoires dans la mucoviscidose sont Staphylococcus aureus, Haemophilus influen$z a e$ et Pseudomonas aeruginosa. L'évolution de l'atteinte respiratoire est toujours progressive, mais la vitesse de cette progression dépend de nombreux facteurs (tableau 2). La survenue d'une infection chronique à Pseudomonas s'accompagne habituellement d'une accélération du déclin de la fonction respiratoire [33]. Ce déclin est moindre chez l'ensemble des patients dont la fonction pancréatique est préservée [34]. Les facteurs influençant la progression de la maladie sont notamment le sexe, des gènes modificateurs, l'exposition à la fumée de tabac, un médiocre statut nutritionnel et l'intensité du traitement [Cutting, pp. 112-116].

L'évolution clinique se caractérise par des exacerbations épisodiques de l'infection respiratoire. Un accroissement de la toux est le plus souvent le premier signe souvent sous-estimé d'exacerbation. Une diminution de l'appétit et de la tolérance à l'effort, de nouveaux râles crépitants à l'auscultation, de nouveaux infiltrats à la radiographie pulmonaire et un déclin de la fonction respiratoire sont d'autres signes et symptômes possibles [35]. Les patients sont le plus souvent apyrétiques et plusieurs de ces troubles sont souvent absents. Les exacerbations tendent à survenir à la suite d'une infection virale [36]. Tandis que la maladie progresse, la toux et l'expectoration augmentent et la capacité d'effort devient de plus en plus limitée.

Les explorations fonctionnelles respiratoires sont initialement normales, mais une obstruction bronchique périphérique est bientôt détectée. Le volume expiratoire maximal par seconde (VEMS) est la variable la plus souvent utilisée pour décrire la progression de la maladie [37]. Au stade terminal, une association d'obstructions et de restrictions et une hypoxie surviennent.

Une hyperinflation est la première anomalie apparaissant à la radiographie pulmonaire, bientôt suivie d'une rétention de mucus, typiquement dans les deux lobes supérieurs. Ces anomalies progressent vers une accentuation des opacités linéaires, une dilatation des bronches, des infiltrats, des kystes et un cour pulmonaire. Des scores permettent de quantifier ces altérations [38].

Diagnostic et aspects cliniques
Tableau 2. Facteurs dont l'influence sur la progression de la maladie est connue
Facteurs constants
Génotype
Sexe
Statut pancréatique
Polymorphisme et gènes modificateurs de la maladie, par ex. TGF- $\beta$, lectines liant le mannose

Facteurs potentiellement modifiables

Age au diagnostic

Colonisation chronique par $P$. aeruginosa ou B. cepacia

Statut nutritionnel médiocre

Condition physique médiocre

Centre de soins de la mucoviscidose

Intensité du traitement et du suivi

Exposition à la fumée de tabac

Observance du traitement

Un certain degré d'hyperréactivité bronchique est présent chez de nombreux patients. Une sensibilisation allergique à l'antigène d'Aspergillus survient chez de nombreux patients mais, avec la progression de la maladie, le syndrome complet d'aspergillose bronchopulmonaire n'est observé que chez $10 \%$ des patients [39]. Des hémoptysies mineures et majeures et un pneumothorax sont des complications apparaissant aux stades avancés de la maladie. Une insuffisance respiratoire survient par la suite, suivie du décès, sauf si une transplantation pulmonaire peut être réalisée.

\section{Atteinte des voies aériennes supérieures}

Des signes radiographiques de pansinusite sont présents chez la quasi-totalité des patients, mais une sinusite symptomatique est peu fréquente. Des polypes nasaux apparaissent fréquemment. Ils peuvent être la cause d'une obstruction nasale gênante et nécessiter une résection [40].

\section{Pancréas}

L'insuffisance pancréatique est la principale cause de retard de la croissance staturo-pondérale chez les patients atteints de mucoviscidose. Elle apparaît chez environ $90 \%$ des patients et est habituellement présente vers l'âge d'un an [7]. Une stéatorrhée, des flatulences, des douleurs abdominales, des pleurs excessifs et une augmentation de l'appétit sont des manifestations typiques chez des patients non traités. La malabsorption des graisses prédomine. La non observance de la prise d'enzymes pancréa- 
tiques demeure la principale cause de douleurs abdominales chez les patients mucoviscidosiques. Certains génotypes sont associés à une meilleure préservation de la fonction pancréatique. Ces patients sont davantage sujets à des épisodes de pancréatite aiguë à l'adolescence et à l'âge adulte [41]. Une intolérance relative au glucose s'accroît avec l'âge, et un diabète lié à la mucoviscidose apparaît chez environ $20 \%$ des adultes jeunes. Un diabète est associé à une évolution plus sévère de la maladie avant et après son diagnostic [42].

\section{Intestin}

Une obstruction de l'intestin grêle distal par du méconium visqueux aboutit à un iléus méconial chez environ $15 \%$ des nouveau-nés atteints d'une mucoviscidose [4]. Au-delà de la période néonatale, tous les patients mucoviscidosiques demeurent sensibles à des épisodes similaires d'occlusion intestinale, qualifiés de syndrome d'occlusion intestinale distale [43]. Des crampes abdominales et une diminution de la production de selles sont les signes initiaux. S'ils ne sont pas reconnus rapidement, ils peuvent progresser vers une occlusion intestinale totale avec distension abdominale et vomissements. L'évolution d'une appendicite est atypique chez les patients mucoviscidosiques et peut aboutir à un abcès du quadrant inférieur droit de l'abdomen, secondaire à une perforation silencieuse [44]. Une invagination est plus fréquente. Un prolapsus rectal récurrent survient chez certains nourrissons atteints d'une mucoviscidose avec malabsorption, le plus souvent avant le début du traitement.

\section{Voies hépatobiliaires}

Une cholestase néonatale peut aboutir au diagnostic de mucoviscidose, mais elle n'est pas associée à une hépatopathie plus tard dans la vie [5]. Une élévation asymptomatique des enzymes hépatiques survient chez de nombreux patients atteints d'une mucoviscidose. Quelquesuns présentent une cirrhose biliaire grave nécessitant une transplantation hépatique ou décèdent de complications d'une hypertension portale et d'un hypersplénisme [45]. Des signes cliniques tels qu'une splénomégalie et une hépatomégalie palpables avec rebord hépatique ferme suggèrent une hépatopathie. Une biopsie du foie procure les informations les plus fiables, mais n'est pas fréquemment effectuée en raison de son aspect invasif. Dans la plupart des centres, une échographie hépatique est effectuée une fois par an afin de déterminer le score de Westaby [46] et de pouvoir ainsi déceler précocement une hépatopathie significative.

\section{Esophage}

Un reflux gastro-œsophagien est souvent présent chez les nourrissons atteints d'une mucoviscidose [47] et une kinésithérapie respiratoire en position de Trendelenburg peut l'aggraver. Ce reflux est également fréquent chez les patients mucoviscidosiques plus âgés, car l'hyperinflation, la toux et l'accroissement des efforts inspiratoires le facilitent.

\section{Nutrition}

Tandis que la maladie progresse, le statut nutritionnel des patients mucoviscidosiques tend à s'aggraver: ils peuvent être hypotrophiques et de faible poids, et leur maturation sexuelle peut être retardée. La préservation d'une croissance staturo-pondérale normale justifie d'importants efforts. Les patients atteints d'une mucoviscidose souffrent d'une malabsorption, leurs besoins caloriques sont plus élevés afin de combattre l'infection chronique, et leur activité métabolique peut être intrinsèquement plus élevée [48]. Ils sont sujets à une carence en vitamines liposolubles, en oligo-éléments et en acides gras essentiels [49]. Leur métabolisme des acides gras essentiel est anormal [50; Strandvik, p. 132].

\section{Autres manifestations}

Un retard de la maturation sexuelle est plus fréquent. Les hommes sont presque toujours stériles. La fertilité est altérée chez les femmes, et une grossesse peut être une menace pour leur santé, particulièrement quand leur fonction respiratoire est compromise [51].

Une perte excessive de sel par la sueur peut aboutir à une déshydratation aiguë [9]. Des apports faibles en sel à certains moments associés à des pertes salines excessives par temps chaud peuvent aboutir à une alcalose métabolique chez les nourrissons mucoviscidosiques [9].

Un cœur pulmonaire est une conséquence d'une atteinte respiratoire avancée et d'une hypoxie prolongée.

Un hippocratisme digital est présent chez la plupart des patients présentant une atteinte respiratoire ou hépatique avancée. Une ostéoporose apparaît chez des adultes jeunes [52]. Avec les traitements plus intensifs de l'infection chronique, l'ostéoarthropathie hypertrophique typique est devenue rare.

\section{Troubles psychologiques}

Un nombre croissant de personnes atteintes de mucoviscidose mène une vie active fructueuse malgré leur maladie et les contraintes liées à son traitement. Pourtant, une santé fragile et un futur incertain exposent un grand nombre d'entre elles à des troubles affectifs et psycholo- 
giques. Trouver un emploi, un compagnon pour la vie et participer au «courant de la vie» n'est pas facile pour les personnes qui souffrent d'une maladie réduisant la durée de l'existence. Tandis que le patient vieillit, de nouvelles complications s'ajoutent, et la complexité et le coût du traitement ne cessent d'augmenter. Souvent pourtant, le poids psychologique perçu n'est pas proportionnel à l'état général objectif d'une personne: une dépression peut survenir chez des patients qui, selon leurs médecins, vont parfaitement bien $[53,54]$.

Les patients mucoviscidosiques ainsi que leurs frères et sœurs sont exposés à un risque accru de troubles psychologiques en raison du stress affectif, des hospitalisations, des charges financières, du temps nécessaire aux soins de l'enfant malade et d'un sentiment d'abandon.

\section{Mucoviscidose atypique ou non classique}

Des patients atteints de mucoviscidose mais dont la fonction pancréatique était préservée étaient déjà identifiés voici de nombreuses années, indiquant la variabilité du spectre de la maladie. Avec la découverte du gène $C F T R$, il est devenu de plus en plus évident que les troubles caractéristiques apparaissant au début de la vie et associés à une mucoviscidose classique ne représentent que le sommet de l'iceberg de la totalité des manifestations pouvant être associées à un dysfonctionnement de la protéine CFTR. Chez certains patients, la symptomatologie est très discrète et la maladie n'apparaît qu'à l'adolescence ou à l'âge adulte. Chez d'autres, une seule caractéristique clinique est présente, par exemple pancréatite récurrente, cholangite sclérosante, bronchectasies «idiopathiques» ou infertilité masculine. Ces deux dernières catégories ont été qualifiées de mucoviscidose atypique ou non classique. Dans la plupart des cas, la concentration des ions chlorure dans la sueur est équivoque (30 à $60 \mathrm{mEq} / \mathrm{l})$. La déclaration américaine de consensus de Rosenstein et Cutting [29] a redéfini les critères diagnostiques de la mucoviscidose à la lumière de ces connaissances récentes. Ces auteurs ont indiqué qu'un diagnostic de mucoviscidose était retenu chez les patients qui présentaient une ou plusieurs caractéristiques phénotypiques ou dont un membre de la fratrie était atteint d'une mucoviscidose ou pour lesquels le résultat du test néonatal de dépistage était positif, plus des données d'examen démontrant une anomalie de la protéine CFTR par un test de la sueur nettement positif ou des mutations des deux allèles du gène CFTR conférant la maladie ou une mesure anormale de la ddp nasale [29]. Cette définition ne différencie cependant pas les patients atteints d'une mucoviscidose classique de ceux chez lesquels le phénotype est moins sévère. Les auteurs d'une récente déclaration européenne de consensus ont été plus pragmatiques: le terme de mucoviscidose classique est réservé aux patients présentant des manifestations cliniques et un test de la sueur positif, tandis que celui de mucoviscidose atypique ou non classique l'est aux patients chez lesquels le test de la sueur est équivoque ou normal, et le diagnostic est conforté par des moyens diagnostiques plus récents, montrant des mutations des deux allèles conférant la maladie ou une anomalie de la ddp nasale [55]. Cette classification est utile mais ne doit pas être appliquée de façon trop stricte. Le phénotype d'une mucoviscidose est réellement un continuum et les catégories se recoupent.

L'étiquette diagnostique mucoviscidose, qui décrit une maladie réduisant l'espérance de vie, comporte une forte charge émotionnelle et peut désavantager le patient, par exemple pour l'obtention d'un emploi ou d'une assurance. Il faut donc souligner que les patients dont la présentation est atypique ne sont pas atteints d'une mucoviscidose classique. Il est encore plus adéquat de définir et de classer les patients présentant une seule atteinte viscérale par la manifestation pathologique elle-même et non par le terme de mucoviscidose atypique, en utilisant par exemple «infertilité par absence bilatérale des canaux déférents» ou "cholangite sclérosante» ou un autre élément de la liste des diagnostics de l'OMS [56]. Le suivi de ces patients est nécessaire pour deux raisons: premièrement, les caractéristiques cliniques peuvent se modifier avec le temps chez un patient donné (par exemple, des infections respiratoires peuvent apparaître chez un sujet présentant une absence bilatérale congénitale des canaux déférents) et deuxièmement, les cliniciens doivent étendre et partager leurs connaissances quant à ce que signifie un dysfonctionnement de la protéine CFTR sur l'ensemble de la vie d'un patient.

Les patients chez lesquels le test de la sueur n'est pas déterminant, porteurs d'une seule mutation identifiée et dont la ddp nasale n'est pas concluante posent le véritable problème diagnostique: ils ne peuvent être classés à l'heure actuelle, car un dysfonctionnement de la protéine CFTR n'a pas été nettement démontré. Ils sont au moins porteurs du trait mucoviscidose. En présence de troubles persistants, ils nécessitent un suivi structuré par un centre adéquat ainsi qu'un traitement symptomatique. Le conseil génétique est important pour ces patients et leurs familles. Il est nécessaire de recueillir des informations à long terme sur ce type de patients, car une amélioration des connaissances s'ensuivra probablement: il est possible, par exemple, que l'association d'une mutation et de plusieurs gènes modificateurs défavorables soit mise en évidence [55]. 


\section{Conseil}

Du fait de la complexité des facteurs affectifs impliqués et du risque de mauvaise compréhension, d'interprétation excessive ou de mémorisation incomplète d'informations pourtant correctement données aux familles et aux parents, le conseil doit être donné par un généticien clinicien. Il est également avantageux de dissocier le conseil de la famille et les soins du patient.

\section{Parents de patients atteints d'une mucoviscidose}

Les parents des enfants atteints de mucoviscidose sont des hétérozygotes obligatoires. Pour chaque grossesse consécutive issue de ces parents, le risque d'atteinte de l'enfant est de $25 \%$. Si les parents désirent d'autres enfants, ils doivent avoir accès à un test prénatal. Quand deux mutations sont connues, des analyses de mutations sont effectuées sur des cellules fotales obtenues par prélèvement de villosités choriales ou amniocentèse [57]. Quand une seule mutation conférant la mucoviscidose a été identifiée, un test $\mathrm{ADN}$ supplémentaire n'est pas obligatoire: un diagnostic prénatal peut également être effectué par analyse de ségrégation des polymorphismes du gène CFTR ou liés à celui-ci. En raison du stress affectif associé à la décision d'interrompre une grossesse, de nombreux patients préfèrent renoncer à avoir d'autres enfants $[58,59]$.

\section{Fratries des patients mucoviscidosiques}

Les manifestations de la mucoviscidose peuvent être très variables, y compris dans une même famille. Une mucoviscidose doit donc toujours être exclue dans la fratrie, non sur des bases cliniques, mais par test formel. Pour les jeunes frères et sœurs, il est conseillé d'exclure une mucoviscidose par le test de la sueur plutôt que par analyse des mutations. Il n'est pas souhaitable de savoir si un mineur est ou non porteur; il est uniquement important de le savoir chez les membres de la fratrie dans le cadre de décisions concernant la procréation. Les parents pourraient surprotéger ou autrement discriminer les porteurs, ce qui n'est pas souhaitable [60].

\section{Membres de la famille des patients atteints d'une mucoviscidose}

Les membres de la famille des patients atteints d'une mucoviscidose sont exposés à un risque accru d'être porteurs d'une mutation du gène CFTR et doivent en être informés. Certains généticiens conseillent d'informer les couples sur les risques qu'ils courent plutôt que les sujets individuellement [61], ce qui est cependant parfois complexe, notamment en cas de changement de partenaire sexuel. La non-détection de l'état de porteur chez un sujet réduit le risque de mucoviscidose chez l'un de ses enfants, mais ne l'exclut pas totalement: les tests de dépistage ne portent que sur les mutations les plus prévalentes ainsi que, si elles ne font pas partie de ces dernières, sur celles exprimées chez les patients mucoviscidosiques.

\section{Conclusion}

La mucoviscidose est une maladie multiviscérale complexe due à un dysfonctionnement de la protéine CFTR .

Dans la grande majorité des cas, cette protéine est totalement non fonctionnelle, et le diagnostic de mucoviscidose est sans ambiguité: le tableau clinique est manifeste et le diagnostic porté sur la base de la symptomatologie est confirmé par le test de la sueur. Une mutation fréquente conférant la maladie est habituellement identifiée sur les deux allèles du gène CFTR. Chez quelques patients, le tableau clinique est beaucoup moins sévère ou se limite même à des troubles affectant un seul organe. Dans ces cas, la mucoviscidose est qualifiée d'atypique. Dans la plupart des cas, des analyses des mutations du gène CFTR et des déterminations biologiques de la fonction de la protéine CFTR confirmeront le diagnostic. En raison de leur rareté, il n'existe aucune preuve démontrant que les mutations de CFTR détectées chez ces patients puissent réellement conférer la maladie. Les connaissances concernant le pronostic à long terme dans ces cas atypiques sont insuffisantes. Ces patients ne sont pas atteints d'une mucoviscidose classique et doivent en être clairement informés.

De nombreux troubles différents peuvent être la première manifestation d'une mucoviscidose, les plus fréquents étant une toux chronique et des selles molles chez un enfant. Un degré élevé de suspicion est le meilleur moyen de ne pas ignorer le diagnostic, particulièrement dans les cas les moins sévères. Le test de la sueur par iontophorèse à la pilocarpine demeure l'examen de choix. La concentration des ions chlorure dans la sueur est supérieure à $60 \mathrm{mmol} / \mathrm{l} \mathrm{chez} \mathrm{les} \mathrm{patients} \mathrm{atteints} \mathrm{d'une} \mathrm{muco-}$ viscidose. Ce test est simple, mais doit être effectué par un laboratoire expérimenté afin que sa précision diagnostique soit satisfaisante. Dans les cas difficiles, les analyses du gène CFTR et les examens biologiques de la fonction de la protéine CFTR apportent une aide supplémentaire. Un diagnostic précoce est nécessaire afin de pouvoir ralentir la progression de la maladie. 
Les infections bactériennes sinusiennes et respiratoires chroniques progressives avec exacerbations intermittentes sont les manifestations les plus typiques de la maladie. Une insuffisance pancréatique est présente chez 90\% des patients. Les hommes sont stériles. Au cours de leur vie, les patients mucoviscidosiques sont exposés à un risque de manifestations pathologiques et de complications atteignant des organes très divers: sinus, poumons, pancréas, foie, intestin, œsophage, os, articulations, etc. Chaque patient est unique en raison de l'association complexe des manifestations possibles de la maladie. Le nom- bre des complications tend à s'accroître avec l'âge; par exemple, $20 \%$ des patients adultes jeunes sont atteints d'un diabète lié à la mucoviscidose.

La mucoviscidose classique est une affection qui réduit l'espérance de vie et, même avec un traitement et un suivi des plus intensifs, la survie médiane actuelle est d'environ 34 ans, ce qui soumet les patients et leurs familles à un stress psychologique extrêmement important.

Les actions de conseil destinées à prévenir la réapparition de la maladie chez des frères et sœurs et dans la famille étendue sont importantes mais complexes.

\section{Références}

1 Report of a Joint Meeting of WHO/ECFTN/ 12 Grosse SD, Boyle CA, Botkin JR, et al: NewICF/(M)A/ECFS: The Molecular Genetic Epidemiology of Cystic Fibrosis. Geneva, WHO, 2006. http//:www.who.int/genomics/ publications/en/

-2 Boucher RC: Regulation of airway surface liquid volume by human airway epithelia. Pflugers Arch 2003;445:495-498.

3 Rosenstein BJ: Diagnostic methods; in Hodson M, Geddes D (eds): Cystic Fibrosis. London, Arnold, 2006, vol 2, pp 177-188.

4 Mushtaq I, Wright VM, Drake DP, et al: Meconium ileus secondary to cystic fibrosis. The East London experience. Pediatr Surg Int 1998;13:365-369.

5 Shapira R, Hadzic N, Francavilla R, et al: Retrospective review of cystic fibrosis presenting as infantile liver disease. Arch Dis Child 1999;81:125-128.

6 Lai HJ, Cheng Y, Farrell PM: The survival advantage of patients with cystic fibrosis diagnosed through neonatal screening: evidence from the United States Cystic Fibrosis Foundation registry data. J Pediatr 2005; 147 : S57-S63.

7 Waters DL, Dorney SF, Gaskin KJ, et al: Pancreatic function in infants identified as having cystic fibrosis in a neonatal screening program. N Engl J Med 1990;322:303-308.

$\checkmark 8$ Abman SH, Accurso FJ, Butler-Simon N, et al: Role of respiratory syncytial virus in early hospitalizations for respiratory distress of young infants with cystic fibrosis. J Pediatr 1988;113:826-830.

9 Sojo A, Rodriguez-Soriano J, Vitoria JC, et al: Chloride deficiency as a presentation or complication of cystic fibrosis. Eur J Pediatr 1994;153:825-828.

10 Chillon M, Casals T, Mercier B, et al: Mutations in the cystic fibrosis gene in patients with congenital absence of the vas deferens. N Engl J Med 1995;332:1475-1480.

11 Muniz AE, Bartle S, Foster R: Edema, anemia, hypoproteinemia, and acrodermatitis enteropathica: an uncommon initial presentation of cystic fibrosis. Pediatr Emerg Care 2004;20:112-114. born screening for cystic fibrosis: evaluation of benefits and risks and recommendations for state newborn screening programs. MMWR Recomm Rep 2004;53:1-36.

13 Scotet V, de Braekeleer M, Roussey M, et al: Neonatal screening for cystic fibrosis in Brittany, France: assessment of 10 years' experience and impact on prenatal diagnosis. Lancet 2000;356:789-794.

14 Gibson LE, Cooke RE: A test for concentration of electrolytes in sweat in cystic fibrosis of the pancreas utilizing pilocarpine by iontophoresis. Pediatrics 1959;23:545-549.

15 Orenstein DM, Winnie GB, Altman H: Cystic fibrosis: a 2002 update. J Pediatr 2002; 140:156-164.

16 LeGrys VA: Sweat testing for the diagnosis of cystic fibrosis: practical considerations. J Pediatr 1996;129:892-897.

17 Guidelines for the performance of the sweat test for the investigation of cystic fibrosis in the UK. Report from the multi-disciplinary working group. July 2002 (http//:www.acb. org.uk).

18 Di Sant'Agnese PA, Darling RC, Perera GA, Shea E: Abnormal electrolyte composition of sweat in cystic fibrosis of the pancreas; clinical significance and relationship to the disease. Pediatrics 1953;12:549-563.

19 Kirk JM, Keston M, McIntosh I, Essa S: Variation of sweat sodium and chloride with age in cystic fibrosis and normal populations: further investigations in equivocal cases. Ann Clin Biochem 1992;29:145-152.

20 Lebecque P, Leal T, De Boeck C, et al: Mutations of the cystic fibrosis gene and intermediate sweat chloride levels in children. Am J Respir Crit Care Med 2002;165:757-761.

21 National Committee for Clinical Laboratory Standards (NCCLS): Sweat Testing: Sample Collection and Quantitative Analysis. Approved guideline C34-A2. Wayne, NCCLS, 2006.
22 Highsmith WE, Burch LH, Zhou Z, et al: A novel mutation in the cystic fibrosis gene in patients with pulmonary disease but normal sweat chloride concentrations. N Engl J Med 1994;331:974-980.

23 Cystic Fibrosis Genotype-Phenotype Consortium: Correlation between genotype and phenotype in patients with cystic fibrosis. N Engl J Med 1993;329:1308-1313.

$24 \mathrm{http}$ //genet.sickkids.on.ca/cgi-bin/WebObjects/MUTATION

25 Schaedel C, Hjelte L, de Monestrol I, et al: Three common CFTR mutations should be included in a neonatal screening programme for cystic fibrosis in Sweden. Clin Genet 1999;56:318-322.

26 Dequeker E, Cuppens H, Dodge J, et al: Recommendations for quality improvement in genetic testing for cystic fibrosis. European Concerted Action on Cystic Fibrosis. Eur J Hum Genet 2000;8(suppl 2):S2-S24.

27 Donaldson SH, Boucher RC: Update on pathogenesis of cystic fibrosis lung disease. Curr Opin Pulm Med 2003;9:486-491.

28 Knowles MR, Paradiso AM, Boucher RC: In vivo nasal potential difference: techniques and protocols for assessing efficacy of gene transfer in cystic fibrosis. Hum Gene Ther 1995;6:445-455.

29 Rosenstein BJ, Cutting GR: The diagnosis of cystic fibrosis: a consensus statement. Cystic Fibrosis Foundation Consensus Panel. J Pediatr 1998;132:589-595.

30 Schuler D, Sermet-Gaudelus I, Wilschanski $\mathrm{M}$, et al: Basic protocol for transepithelial nasal potential difference measurements. J Cyst Fibros 2004;3(suppl 2):151-155.

31 Standaert TA, Boitano L, Emerson J, et al: Standardized procedure for measurement of nasal potential difference: an outcome measure in multicenter cystic fibrosis clinical trials. Pediatr Pulmonol 2004;37:385-392.

-32 Armstrong DS, Grimwood K, Carlin JB, et al: Lower airway inflammation in infants and young children with cystic fibrosis. Am J Respir Crit Care Med 1997;156:1197-1204. 
-33 Frederiksen B, Koch C, Hoiby N: Antibiotic treatment of initial colonization with Pseudomonas aeruginosa postpones chronic infection and prevents deterioration of pulmonary function in cystic fibrosis. Pediatr Pulmonol 1997;23:330-335.

-34 Corey M, Edwards L, Levison H, Knowles M: Longitudinal analysis of pulmonary function decline in patients with cystic fibrosis. J Pediatr 1997;131:809-814.

- 35 Rosenfeld M, Emerson J, Williams-Warren J, et al: Defining a pulmonary exacerbation in cystic fibrosis. J Pediatr 2001;139:359-365.

- 36 Collinson J, Nicholson KG, Cancio E, et al: Effects of upper respiratory tract infections in patients with cystic fibrosis. Thorax 1996; 51:1115-1122.

- 37 Corey M, McLaughlin FJ, Williams M, Levison $\mathrm{H}$ : A comparison of survival, growth, and pulmonary function in patients with cystic fibrosis in Boston and Toronto. J Clin Epidemiol 1988;41:583-591.

38 Brasfield D, Hicks G, Soong S, Tiller RE: The chest roentgenogram in cystic fibrosis: a new scoring system. Pediatrics 1979;63:24-29.

-39 Mastella G, Rainisio M, Harms HK, et al: Allergic bronchopulmonary aspergillosis in cystic fibrosis. Eur Respir J 2001;17:10521053.

40 Jorissen MB, De Boeck K, Cuppens H: Genotype-phenotype correlations for the paranasal sinuses in cystic fibrosis. Am J Respir Crit Care Med 1999;159:1412-1416.

41 De Boeck K, Weren M, Proesmans M, Kerem E: Pancreatitis among patients with cystic fibrosis: correlation with pancreatic status and genotype. Pediatrics 2005; 115:e463e469.
42 Moran A, Hardin D, Rodman D, et al: Diagnosis, screening and management of cystic fibrosis related diabetes mellitus: a consensus conference report. Diabetes Res Clin Pract 1999;45:61-73.

43 Mascarenhas MR: Treatment of gastrointestinal problems in cystic fibrosis. Curr Treat Options Gastroenterol 2003;6:427-441.

44 Martens M, De Boeck K, Van Der Steen K, et al: A right lower quadrant mass in cystic fibrosis: a diagnostic challenge. Eur J Pediatr 1992;151:329-331.

45 Sokol RJ, Durie PR: Recommendations for management of liver and biliary tract disease in cystic fibrosis. Cystic Fibrosis Foundation Hepatobiliary Disease Consensus Group. J Pediatr Gastroenterol Nutr 1999;28(suppl 1):S1-S13

46 Williams SG, Evanson JE, Barrett N, et al: An ultrasound scoring system for the diagnosis of liver disease in cystic fibrosis. J Hepatol 1995;22:513-521.

47 Heine RG, Button BM, Olinsky A, et al: Gastro-oesophageal reflux in infants under 6 months with cystic fibrosis. Arch Dis Child 1998;78:44-48.

48 Sinaasappel M, Stern M, Littlewood J, et al: Nutrition in patients with cystic fibrosis: a European Consensus. J Cyst Fibros 2002;1: 51-75.

49 Roulet M, Frascarolo P, Rappaz I, Pilet M: Essential fatty acid deficiency in well nourished young cystic fibrosis patients. Eur J Pediatr 1997;156:952-956.
50 Strandvik B, Gronowitz E, Enlund F, et al: Essential fatty acid deficiency in relation to genotype in patients with cystic fibrosis. J Pediatr 2001;139:650-655.

- 51 Edenborough FP, Stableforth DE, Webb AK, et al: Outcome of pregnancy in women with cystic fibrosis. Thorax 1995;50:170-174.

52 Conway S: Osteoporosis is cystic fibrosis. J Cyst Fibros 2003;2:161-162.

53 Elgudin L, Kishan S, Howe D: Depression in children and adolescents with cystic fibrosis: case studies. Int J Psychiatry Med 2004;34: 391-397.

54 Kotwicki RJ, Condra L, Vermeulen L, et al: Assessing the quality of life in children with cystic fibrosis. WMJ 2001;100:50-54.

55 De Boeck K, Wilschanski M, Castellani C, et al: Cystic fibrosis: terminology and diagnostic algorithms. Thorax 2005.

56 Classification of cystic fibrosis and related disorders. J Cyst Fibros 2002;1:5-8

57 Richards CS, Grody WW: Prenatal screening for cystic fibrosis: past, present and future. Expert Rev Mol Diagn 2004;4:49-62.

58 Evers-Kiebooms G, Denayer L, Cassiman JJ, Van den Berghe H: Family planning decisions after the birth of a cystic fibrosis child: the impact of prenatal diagnosis. Scand J Gastroenterol 1988;23:38-46.

59 Mischler EH, Wilfond BS, Fost N, et al: Cystic fibrosis newborn screening: impact on reproductive behavior and implications for genetic counseling. Pediatrics 1998;102:4452.

60 Clarke A: The genetic testing of children. Working Party of the Clinical Genetics Society (UK). J Med Genet 1994;31:785-797.

-61 Super M, Schwarz MJ, Malone G, et al: Active cascade testing for carriers of cystic fibrosis gene. BMJ 1994;308:1462-1467. 